\title{
Investigación acción en educación, interrogantes y respuestas
}

\author{
Eunice Sequea Romero ${ }^{1}$
}

\section{Introducción}

El interés por el tema de la docencia reflexiva y el docente como investigador han llevado a la autora de este artículo a la búsqueda permanente de fundamentos y métodos para articular la teoría y la práctica, lo que ha sido posible en el contexto de una maestría dedicada a la función docente en Educación Superior. La utilización de la investigación acción, tanto en los proyectos personales, como en aquellos realizados por los estudiantes en mi función como tutora o asesora han permitido la reflexión sobre los alcances y las potencialidades de la investigación acción y también sobre su vigencia. El artículo pretende dar respuesta a algunas de las interrogantes que se hacen los investigadores noveles cuando seleccionan esta alternativa metodológica para sus proyectos .en organizaciones educativas

\section{1. ¿Por qué seleccionar la investigación acción para la formación y actualización docente?}

Es importante recordar que el término de investigación acción se le atribuye a Kurt Lewin (1944). Al analizar los objetivos de investigación planteó dos clases de investigación científica: la de las leyes generales y otra enfocada en la acción con una metodología que abarcaba recolección de información, conceptualización, planeación, ejecución y evaluación, pasos que podían repetirse

Al revisar los antecedentes históricos de la investigación acción en el aula, también llamada investigación del profesor, es necesario iniciar con el trabajo de Stenhouse (1920-1969) quien plantea la idea de un movimiento conocido como profesores investigadores en

$1 \quad$ Doctora en Sociología de la Educación en la Sorbona de París, Diploma de Estudios Avanzados en Psicología y Educación en la Universidad Paris 8. Docente en el Doctorado en Educación y la Maestría de Docencia para Educación Superior en la Universidad Nacional Experimental Rafael María Baralt 
el Centro para la Investigación Aplicada a la Educación (Centre for applied Research in Education, CARE) de la Universidad East Anglia en Reino Unido.

El uso de la investigación acción en la exploración y búsqueda de cambio en el contexto educativo, tales como implementación de reformas educativas, revisión de la práctica de los docentes, mejoramiento de los centros educativos de acuerdo con las orientaciones y demandas sociales e institucionales puede ubicarse con el proyecto Humanities Project, liderado por Stenhouse (19201969) en 1967 en Gran Bretaña. La propuesta se enfoca en la reflexión, la práctica y el cambio en el contexto de los centros educativos (el aula y la institución).

La idea de una investigación realizada por los docentes, sobre su práctica, desafiaba el paradigma de investigación dominante en la primera mitad del S. XX, enfocado en la causalidad, que estudiaba los problemas a través de modelos lineales y deterministas, se desarrolló una investigación sobre la educación cuyos conocimientos provienen mayoritariamente de la psicología y la sociología. El método comprendía observación de conductas y deducción de relaciones causales entre variables ejemplo: estímulos de parte del profesor y cambios en la conducta del aprendiz.

De la sociología norteamericana surge que los hechos sociales causaban otros hechos como un encadenamiento mecánico, se estudiaba a través de las correlaciones. Las metáforas que dominaron la investigación educacional desde los 50 a los 70 fueron el aula como la caja de Skinner, y los sistemas escolares y la sociedad como partes eslabonadas de una gran máquina disociadas entre sí.

A partir de la crítica al currículo por objetivos, dominante durante la década de los 60, Stenhouse (1991) plantea el currículo como un proyecto en desarrollo, que necesita de la confrontación con la práctica, de la participación activa de los encargados de su implementación, de manera especial los docentes, quienes deberían pasar de seguir pasivamente las prescripciones curriculares a convertirse en investigadores de su aula y mantener una actitud crítica 
y contextualizada en la enseñanza de los contenidos del currículo y de las teorías en las que se soportan.

Esta concepción trasciende la visión de un currículo rígido y centralizado, en su lugar plantea dos acepciones: como intención (ideas, principios y fines) y como realidad (operatividad, concreción). Como intención debe permanecer abierto a la crítica, como realidad debe permitir el desarrollo profesional del docente "ayudar al profesor a fortalecer su práctica comprobando de modo sistemático método e ideas" (Stenhouse, 1991).

La investigación y el desarrollo del currículo corresponden al docente. Para Stenhouse (1991) cada aula sería una especie de laboratorio que serviría para la validación de contenidos y orientaciones emanadas del currículo oficial. Es una investigación que no busca generar leyes, parte del reconocimiento de la complejidad del proceso que tiene lugar en el aula, no busca medir variables, utiliza la reflexión de la práctica como procedimiento, por tanto, es de naturaleza cualitativa y, finalmente, debe contribuir a la autonomía del docente en relación con su trabajo.

Stenhouse (1996) considera que los docentes deben investigar para mejorar la capacidad de generar conocimientos profesionales, en lugar de solo consumidores del aquel proveniente de los especialistas. Se requiere desarrollar constantemente conocimientos profesionales en relación con circunstancias cambiantes. Es necesario trascender la posición de solo usuario del conocimiento, que es generalizado a partir de muestras, por el contrario, este enfoque (docente investigador) trabaja con estudio de casos. Este modelo generador de conocimientos asume que las mejoras en la práctica parten de la comprensión de los docentes.

Además de Stenhouse es importante revisar los aportes de Elliot en el Reino Unido, quien formó parte del equipo y posteriormente coordinó varios de los proyectos de investigación reseñados en sus obras La investigación-acción en Educación (1994) y El Cambio Educativo desde la Investigación Acción (1993). En estas publicaciones expone las experiencias investigativas que coordinó: 
Ford Teaching Project y Teacher-Student Interaction and Quality of Learning Project.

Junto a la narración del trabajo realizado en estos proyectos, se explicitan sus reflexiones teóricas, epistemológicas y metodológicas relacionadas con la investigación acción. Es una investigación que se ocupa de las acciones humanas y sociales experimentadas por los docentes, y esas acciones deben ser problemáticas, contingentes (sujetas a cambio) y prescriptivas (dar respuestas prácticas).

De acuerdo con los citados autores, la investigación acción se relaciona con los problemas prácticos cotidianos experimentados por los docentes, no por los teóricos. Esta investigación puede ser desarrollada por los propios docentes o alguien que ellos encarguen. Su propósito es profundizar la comprensión de un problema (exploratoria) frente a las definiciones iniciales que se tengan. No impone una respuesta, sino que mejora la comprensión.

La postura teórica que adopta al respecto es que la acción para cambiar requiere una comprensión profunda. Elliot (1994) diferencia la investigación educativa de aquella sobre la educación con base en 8 parámetros: perspectiva, conceptos, datos, teoría, método, generalización, participación en el análisis de los datos y técnicas.

Los objetivos deben derivarse de las tareas propias de la investigación acción, que son los problemas derivados de la puesta en marcha en la práctica de cualquier idea educativa general, es decir, la identificación de lagunas que aparezcan entre las intenciones previstas en los planes y las situaciones prácticas "la investigación acción se dirige a los problemas prácticos que los profesionales experimentan como tales y a las estrategias que pueden emplear para solucionarlos" Elliot (1994, p. 67)

El protagonismo del docente en esta investigación, así como sus fines generaron un creciente interés en la comunidad académica, al punto que en 1976 se crea la red Investigación acción en clase desde el Cambridge Institute of Education, para difundir las ideas de la teoría y la práctica de la investigación acción educativa, y así conectar 
a los sujetos que la utilizaban, tanto en Europa como Estados Unidos y Canadá.

Kemmis y otros investigadores australianos, McTaggart y Grundy, han contribuido a ver esta investigación desde una perspectiva no solo comprensiva, sino también crítica. Kemmis (1993) al referirse al planteamiento de Stenhouse, señala que puso énfasis en la distancia entre las ideas y su operatividad cuando define el currículo como "un intento de comunicar los principios esenciales de una propuesta educativa de tal forma que quede abierta al escrutinio crítico y pueda ser traducida efectivamente a la práctica" (p. 28), de allí que los profesores son actores fundamentales, no operarios en el proceso educativo y la práctica realza la responsabilidad moral de los profesores.

Según Kemmis (1993), éste es un paso hacia su emancipación, pero no suficiente, se deben estudiar también los procesos mediante los cuales se estructura el currículo fuera de la escuela. En esto se centran los teóricos críticos. Es una perspectiva de la profesión como fuente de autocrítica de la teoría y la práctica de quienes la llevan a cabo. De acuerdo con este autor, la teoría práctica anima a reorientar su trabajo en clase y la escuela según sus valores y deliberación práctica, pero poco trata el papel de Estado.

Las reformas educativas de finales del siglo XX enfatizaron la necesidad de un perfil docente más acorde con los cambios ocurridos en la sociedad y con los cambios necesarios para el S. XXI, al respecto Porlan et al. (2001) destacan los siguientes rasgos:

- Conocimiento profesional genuino y actualizado

- Una visión investigadora de su trabajo docente

- Mayor capacidad de reflexión y tomar decisiones de manera autónoma y cooperativa

Estos rasgos en el perfil docente y los desafíos de la sociedad actual desde nuestro punto de vista reafirman la vigencia de la investigación acción entre las alternativas metodológicas disponibles para los docentes investigadores.

\section{2. ¿Cómo comienza la investigación acción y cuando concluye?}


Inicio. Supone una práctica educativa problemática por sus resultados, por la discrepancia entre su idea de origen y su aplicación, por la inconformidad de los actores con respecto al proceso de implementación, pero no es una relación causal determinista, por el contrario es multicausal y compleja, de allí que se requieren una exploración y una sensibilización de los participantes para llegar a la comprensión, lo que llevara a focalizar el tema a través de los consensos entre los participantes.

Quién la inicia. Puede ser parte de un proyecto institucional o también de cooperación interinstitucional, por ejemplo, una universidad que presta asesoría a un instituto de formación, en el cual los docentes desarrollan sus proyectos, también por iniciativa individual, cuando un docente o un equipo emprende el proyecto generan cambios o innovaciones en su práctica educativa, por ejemplo, estudiantes de maestría o doctorado que aplican esta investigación para sus trabajos de grado.

Rasgos que la distinguen

- Participativa. Trabajan en equipo los responsables, los usuarios, los asesores y los prácticos, dependiendo del proyecto.

- Flexibilidad de la investigación. Conlleva establecer acuerdos en cuanto a los objetivos, los medios para lograrlos, control de la información y su divulgación. Al elaborar los planes de acción, es importante considerar este rasgo por cuanto, la puesta en marcha del plan implica la revisión permanente y el afrontar dificultades que deberán resolverse entre todos, situaciones como la realización de una actividad, el acceso a la información o a los recursos y los contextos institucionales con frecuencia llevan a reprogramar el plan general .

- Formativa. Debe ir más allá de acciones que produzcan cambios, debe generar reflexión permanente a lo largo del proceso y promover la autonomía del aprendizaje y el cambio de actitud hacia el trabajo colaborativo. 
- Metacognición y ética. En el caso del investigador debe aclararse en cuanto a los supuestos iniciales sobre el enfoque de investigación que utilizará y también los supuestos sobre la problemática que da origen a la investigación y en el caso de los involucrados (actores o participantes) explicitar también los significados y su origen, lo compartido y cultural. En cuanto a los ético el respeto a los acuerdos (confidencialidad, plan y actividades) y a la percepción del otro.

Culminación. La investigación acción ha sido descrita como una espiral, es decir, un proceso que tiene etapas, ciclos, fases o momentos en la búsqueda de alcanzar los objetivos programados, sin embargo, destaca la circularidad o recursividad, que da la posibilidad de vuelta, de revisión o reformulación de lo planeado, así mismo, los resultados pueden generar nuevas propuestas y en el caso del o de los investigadores responsable de ejecutar (docentes) se promueve la necesidad de revisión permanente de su práctica, es decir, que se cierra el proyecto por necesidades puntuales, mas no las inquietudes de los docentes investigadores y su compromiso con las metas educativas

Lewin propuso la investigación acción como espiral de ciclos que comprende diagnóstico, planificación, desarrollo de las acciones y la evaluación, con flexibilidad para volver sobre ciclos anteriores. La flexibilidad del modelo implica la revisión de la puesta en marcha, lo que puede llevar hasta la planificación, esto se logra a través de la evaluación permanente de las acciones emprendidas. 
Proceso de investigación acción

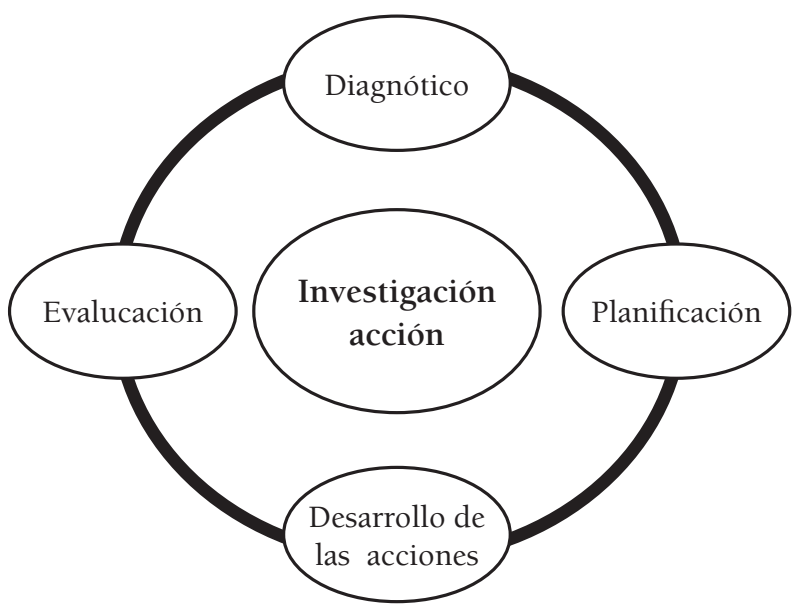

Sequea a partir de la idea de Lewin (1944)

\section{3. ¿Cuáles son los fundamentos teóricos, epistemológicos y metodológicos de la investigación acción en educación?}

La interrogante sobre los fundamentos requiere puntualizar que todo investigador tiene unos supuestos que guían y orientan su práctica en cuanto al método, la selección de técnicas, la teorización y la interpretación.

En el caso de la investigación acción educativa, no hay un solo punto de vista, se fundamenta con base a diferentes criterios, en este artículo se abordaran dos que se apoyan fundamentalmente en los llamados intereses del conocimiento y fundamentos epistemológicos del paradigma cualitativo. El primer criterio parte de los intereses cognitivos básicos.

Cuando Habermas (1990) plantea una teoría de la ciencia llamada a "aprehender sistemáticamente el contexto de utilización y de constitución de las teorías científicas” (p. 19) distingue las de acción instrumental (interés técnico) de las de la comprensibilidad intersubjetiva (interés práctico) y aquellas donde se vincula acciónexperiencia (interés emancipado). Cada uno se relaciona con un tipo de ciencia: empírico-analítico, histórica-hermenéutica y crítica. 
Grundy (1998) basándose en Habermas refiere que hay congruencia entre el interés técnico y las perspectivas de acción de las ciencias empírico-analíticas. Este saber se basa en la experiencia y la observación producto de la experimentación. Las teorías derivadas abarcan conexiones hipotético-deductivas que luego derivan en deducciones de contenido empírico. Como la ciencia empírico-analítica se ocupa de identificar las regularidades que existen en el medio, es posible formular reglas para la acción. Al establecer regularidades es posible la predicción de los fenómenos en circunstancia similares.

\section{Comprensiva e interpretativa}

Es la que se trabajó en los proyectos de Elliot más enfocada en la comprensión, a través de la reflexión de las situaciones conflictivas, tal como se explicó en la interrogante uno. Elliot (1994), dice que la teoría de la comprensión manejada en el CARE se identifica con Gadamer, lo que les ha acarreado la crítica de los emancipadores.

Para Gadamer, citado por Elliot (1994), "las formas de comprensión que tienen como objeto propio las personas dan un conocimiento moral que guía la elección y el juicio de las situaciones prácticas" (p. 115). Gadamer (1975) explica que en la tradición hermenéutica se distinguen la comprensión, la interpretación (explicitación de la comprensión) y la aplicación esta última relegada como parte de esa unidad, sin embargo, él la reivindica y dice que son tres momentos del proceso de comprensión. Además, la aplicación es importante porque es conexión con el contexto "esto es el carácter de la hermenéutica histórica cuando reflexiona sobre la tensión entre identidad del asunto compartido y la situación cambiante en la que trata de entenderse" (p. 194).

El Interés práctico se orienta hacia la comprensión del medio para interactuar en él "recibe el nombre de práctico porque el interés viene dado por realizar la acción correcta en una situación concreta" Grundy (1998, p. 30). Hay tres categorías que destacan en este tipo de interés: la interacción, comunicación entre los participantes, el consenso en el proceso de comprensión e interpretación, son los acuerdos entre los sujetos involucrados y subjetividad, ya que es 
conocimiento humano de los sujetos en acción y no conocimiento de la realidad como objeto.

Grundy (1998) lo define como "interés fundamental para comprender el ambiente mediante interacción, basado en una interpretación consensuada del significado" (p. 32).

\section{Crítica emancipadora}

Para Habermas el interés emancipatorio sería independencia de todo lo que esta fuera del individuo, es posible cuando hay autorreflexión y está ligada a los ideales de justicia e igualdad. "la autorreflexión es a la vez intuición y emancipación comprensión y liberación de dependencias dogmáticas... Solo el que se aprehende a sí mismo como sujeto... logra su autonomía" Habermas, citado por Grundy (1998, p. 36). Este tipo de interés genera teorías críticas, que son aquellas capaces de deconstruir lo evidente o aceptado incondicionalmente.

La teoría crítica pone en el tapete la relación educaciónsociedad, como la escuela sirve a los intereses del estado y su currículo y como el Estado representa a los intereses de la sociedad. Propone formas cooperativas de trabajo entre docentes y otras ciudadanos relacionados con la educación para tener visiones críticas que se opongan a los presupuestos y actividades educativas del Estado, no solo a través de las ideas, sino de las actividades, se trata de llegar a una política educativa práctica.

La teoría crítica del currículo es una forma de razonamiento distinta a la técnica y a la práctica, es el razonamiento dialéctico, nunca se parte de cero y está orientada por un tipo de interés emancipatorio. Parte de una concepción de la realidad compleja y dinámica, que condiciona las acciones de los sujetos y sus percepciones.

La investigación acción propone una relación de sujetos en interacción, ya sea en la búsqueda de la comprensión o de un conocimiento desalienado y emancipador. La interacción debe basarse en un diálogo permanente. 
Desde una perspectiva paradigmática de investigación, se han realizado diferentes comparaciones entre los fundamentos del llamado paradigma cualitativo, que abarca el interpretativo y el crítico, en oposición al empírico analítico, conocido también como cuantitativo que subraya las siguientes diferencias:

Paradigma cuantitativo y cualitativo

\begin{tabular}{|c|c|c|}
\hline Categorías & Cuantitativo & Cualitativo \\
\hline $\begin{array}{l}\text { Naturaleza de la } \\
\text { realidad }\end{array}$ & $\begin{array}{l}\text { Externa al sujeto y es } \\
\text { percibida equilibrada } \\
\text { y compuesta por } \\
\text { regularidades. }\end{array}$ & $\begin{array}{l}\text { Compleja, múltiple y } \\
\text { construida. }\end{array}$ \\
\hline $\begin{array}{l}\text { Relación } \\
\text { investigador } \\
\text { y fenómeno } \\
\text { estudiado }\end{array}$ & $\begin{array}{c}\text { Independencia de uno } \\
\text { con el otro. Garantiza la } \\
\text { objetividad. }\end{array}$ & $\begin{array}{c}\text { Interacción entre } \\
\text { investigador y los } \\
\text { sujetos implicados } \\
\text { en el proceso. } \\
\text { Sujeto cognoscente } \\
\text { inseparable de lo que } \\
\text { conoce. }\end{array}$ \\
\hline $\begin{array}{l}\text { Alcance de la } \\
\text { investigación }\end{array}$ & $\begin{array}{l}\text { Explicación de nexos } \\
\text { causales y búsqueda } \\
\text { de control de variables } \\
\text { para poder generalizar } \\
\text { resultados. }\end{array}$ & $\begin{array}{l}\text { Descripción y } \\
\text { comprensión de la } \\
\text { realidad en estudio. }\end{array}$ \\
\hline Métodos & $\begin{array}{c}\text { Empírico-análitico. Verifica } \\
\text { o refuta con los resultados } \\
\text { teorías de las cuales parte } \\
\text { la investigación }\end{array}$ & $\begin{array}{l}\text { Análisis inductivo } \\
\text { de los datos. } \\
\text { Interpretación } \\
\text { ideográfica que } \\
\text { emerge de las } \\
\text { particularidades } \\
\text { (caso, contexto e } \\
\text { informantes) }\end{array}$ \\
\hline
\end{tabular}

Es importante destacar que cuando la comparación entre paradigmas separa al paradigma interpretativo del crítico, las categorías formuladas en el cuadro, correspondiente al cualitativo son comunes a ambos, solo que la investigación acción educativa busca explícitamente cambios en las prácticas educativas y en la metodología en cuanto a la espiral de etapas y ciclos. 


\section{4. ¿Qué papeles juegan la práctica y la teoría en la investigación acción?}

\section{La práctica}

Desde el planteamiento de Lewin de la investigación acción como un proceso cíclico de exploración, actuación y valoración de resultados, aquellos que la utilizan dan un papel fundamental a la práctica, muchas veces con escaso énfasis en la teoría, por lo que es importante aclarar este aspecto. Autores estudiosos del currículo y la investigación de los docentes, ya citados en las partes precedentes, tales como Stenhouse, Elliot, Kemmis, Carr y Grundy, entre otros, han explicado su concepción de la práctica docente remontándose a Aristóteles, quien hizo una diferencia entre:

Poiesis, como acción orientada a producir un objeto o artefacto.

Aquí predomina un juicio o razonamiento técnico, hay un fines predeterminados y se cumplen reglas ya conocidas. En la poiesis predomina la tekne, que son las técnicas para elaborar algo, estas se pueden aprender, aun en solitario para obtener mayor destreza y maestría.

Praxis, acciones no orientadas a realizarse para alcanzar un fin externo, sino una práctica donde hay un discernimiento ético.

En la segunda predomina el juicio o razonamiento práctico, en griego la phronesis, que es la disposición al bien, la phronesis es social e intersubjetiva porque hay diversidad, es mediadora e imprevisible, como dice Grundy (1998) "opera en cuestiones que tienen como regla general el bien y cuyo resultado es imprevisible". En ella las personas razonan teniendo como base su experiencia sobre el cómo actuar. Aquí median sus valores y las circunstancias históricas contextuales, por eso los medios y los fines son problemáticos.

En el caso de la práctica educativa concebida bajo la visión de praxis, el docente trabaja en un contexto complejo, donde deben someter sus decisiones a deliberación y en ella confluyen los conocimientos de su formación, los provenientes de la experiencia, que en algunos casos contradicen los primeros y su juicio del bien. Frente a un problema o una situación que, por ejemplo, implica una 
decisión entre dos o más opciones, se produce la deliberación, no se trata de aplicar una fórmula, receta o técnica, sino de hacerlo con base en un juicio o razonamiento práctico.

Tradicionalmente, la formación basada en el currículo llamada por objetivos, tecnocrático o tecnológico proporcionó conocimientos, provenientes del experto y de investigaciones realizadas con una metodología hipotética deductiva, la formación implicaba "consumir" y "acumular" para aplicar ese conocimiento, una separación entre teoría y práctica, con valoración de la teoría formal, la segunda depende de la primera.

De acuerdo con Schön (1987) este conocimiento responde a una racionalidad técnica, al separar conocimiento y práctica y al supeditar la segunda a la teoría, la acción consistiría solo en conseguir los medios para alcanzar un fin predeterminado.

Además, señala este autor que los contextos prácticos no son estables, por tanto los problemas no son anticipables. En ese caso el práctico debe revisar y criticar su comprensión, construir una nueva descripción y comprobarla. El conocimiento en acción es de carácter ético más que técnico y como son inestables, no son predecibles.

A partir del llamado currículo por proceso, se hace énfasis en la práctica, en el docente como productor de conocimiento y en la práctica reflexiva, tomando en cuenta el carácter ético de ese conocimiento más la inestabilidad de las situaciones, lo que hace poco predecible la teoría en uso para una situación determinada.

De acuerdo con Lawn (1998), el centro del movimiento del docente investigador es la práctica, la que se desarrolla en clase, ella puede mejorarse con el estudio y la reflexión sistemática "En el proceso de estudio, los maestros perfeccionan su juicio profesional, se responsabilizan y restauran su dignidad. Se liberan" (p. 126).

\section{La teoría}

La investigación en educación ha utilizado teorías provenientes de otros campos del saber, fundamentalmente de la psicología y de la sociología, con base en ellas se han diseñado estrategias para ser aplicadas por los docentes. Las teorías constituyen los marcos 
explicativos de los problemas que se investigan. Cuando desde la investigación acción educativa se hace énfasis en la práctica, es importante hacer algunas consideraciones al respecto.

Elliot (1993) refería que los profesores sienten la teoría como una amenaza, porque está hecha por expertos que pretenden producir conocimientos válidos para su práctica, es una especie de poder externo, esto trae como consecuencia que la teoría se impone y margina el conocimiento profesional proveniente de la experiencia. Además, al ser generalizaciones se desconoce el carácter singular de la práctica "la generalización constituye la negación de la experiencia cotidiana de los prácticos" (p. 136).

Las teorías constituyen marcos explicativos de los fenómenos a los que se refieren, se componen de proposiciones y conceptos con capacidad predictiva, ya que provienen de un método que busca la generalización de resultados, por tanto, hay problemas con su aplicación automática en contextos singulares, no probabilísticos. En el caso de la investigación cualitativa en general y de la investigación acción en particular, se han destacado algunos aspectos como la singularidad de los fenómenos y la no predictibilidad de los resultados por lo caben las preguntas qué papel juegan las teorías formales, puede generarse teoría y cuáles características tiene esa teoría.

La investigación acción educativa busca generar cambios en la práctica, para lograrlo requiere la comprensión de la situación por los involucrados, a través de procesos reflexivos que implicarán: explicitación de las teorías en uso, contrastación de éstas con la práctica y posiblemente generación de nuevas explicaciones, es decir, nuevas formas de aproximación a las teorías, por tanto, hay una apropiación crítica y contextualizada donde se vinculan teoríapráctica.

Metodológicamente, al enfocarse en un aula, un programa o una institución, se le considera un estudio de caso, el cual según Stake (1999) debe abarcar la complejidad de un caso particular. Se busca el detalle de la interacción con sus contextos. "Es el estudio de la particularidad y de la complejidad en un caso singular, para llegar a comprender su actividad en circunstancias importantes" (p. 
17). Este autor los divide en intrínsecos cuando no se elige, sino estamos obligados a tomarlo como caso y hay otros donde es una necesidad general y se estudia a partir de un caso. Con éste se busca comprender algo más allá del caso, son los casos instrumentales, aquí se podrían seleccionar varios casos en lugar de uno.

"El cometido del estudio de caso es la particularización, no la generalización... se llega a conocerlo bien, y no principalmente para ver en qué se diferencian los unos de los otros, sino para ver qué es" (Stake, 1991, p. 20), sin embargo, Hammersley y Atkinson (1994) apoyándose en Glasser y Strauss distinguen entre teorías formales y sustantivas, las últimas se generan en los estudios cualitativos, referidos a casos y no a muestras representativas.

En la teorización sustantiva, según Bogdan y Taylor (1994), el análisis de las categorías analíticas y sus resultados conducen a una abstracción. Esa explicación se compone también de conceptos, en este caso "sensibilizadores" en oposición a los formalistas. Stake (1999) los denomina "universales concretos". Por su parte, Elliot (1994) dice que a partir de estos conceptos sensibilizadores podría generarse una teoría desde la práctica.

Desde una perspectiva crítica existe una metateoría, Kemmis (1993), que subyace en la naturaleza de los problemas prácticos y que destaca:

1. Medios y fines son igualmente problemáticos

2. Los valores se toman en cuenta pero no se determinan porque entran en competencia

3. Se requiere acción meditada y reflexiva, pero no siguiendo reglas, principios o procedimientos establecidos

4. La responsabilidad recae en el actor

La investigación acción educativa se enfoca en los cambios de la práctica realizados por sus actores, a través de procesos reflexivos para alcanzar primero la comprensión de lo que ocurre, eso implica explicitar las teorías en uso y confrontar ese conocimiento con el experiencial. De allí se construye un conocimiento propio y explicaciones o abstracciones que deberían renovar la práctica 
educativa. Como señala Morse (2003) al explicar los procesos cognitivos en el análisis de los datos y la teorización en la investigación cualitativa "la teoría le da estructura a los datos y les da aplicación a los hallazgos" (p. 40).

Finalmente, en la comprensión de la (s) teoría (s) es conveniente recordar lo que explica Gadamer (1975) cuando analiza el proceso hermenéutico y lo relaciona con la ética según Aristóteles, al respecto dice "lo bueno para el hombre solo aparece en la concreción de la situación práctica en la que se encuentra, significa que un saber general que no sepa aplicarse a la situación concreta carecería de sentido" (p. 194).

\section{Reflexiones finales}

Los contextos sociales, económicos y tecnológicos han cambiado mucho desde las propuestas iniciales de la investigación acción educativa, sin embargo, las temáticas abordadas, la metodología utilizada y sus propósitos no han perdido vigencia. Los retos permanentes que en la actualidad deben asumir los docentes en su práctica para contribuir con la formación de generaciones que actuarán en contextos complejos dinámicos y desafiantes propician la necesidad de una docencia reflexiva, que genere conocimientos profesionales, a partir del aprendizaje permanente, la investigación y el compromiso educativo, sobre todo cuando se actúa en sistemas educativos donde las estructuras institucionales y la burocracia limitan las innovaciones.

\section{Referencias}

Bogdan, R., \& Taylor, S. (1994). A positive approach to qualitative evaluation and policy research in social work. Qualitative research in social work, 293-302.

Elliot, J. (1993). El cambio educativo desde la investigación acción. España: Editorial Morata.

Elliot, J. (1994). La investigación acción en educación. España: Editorial Morata. 
Gadamer, H-G. (1975). Verdad y método. Salamanca: Editorial Sígueme.

Grundy, S. (1998). Producto o praxis del curriculum. España: Editorial Morata.

Habermas, J. (1990.) Teoría y praxis. España: Editorial Tecnos.

Hammersley, M., \& Atkinson, P. (1994). Etnografía. España: Editorial Paidós.

Kemmis, S. (1993). El curriculum más allá de las teorías de la reproducción. España: Editorial Morata.

Lawn, M. (1998). Atrapados en las tareas escolares. Las posibilidades de los docentes de investigar en su trabajo en Calidad de la enseñanza e investigación acción. Sevilla: Editorial Diada.

Lewin, K. (1944). The dynamics of group action. Educational leadership, 1(4), 195-200.

Morse, J. (2003). Asuntos críticos en los métodos de investigación cualitativa. Universidad de Antioquia. Colombia

Porlán, R., Martín del Pozo, R., Martín, J., \& Rivero, A. (2001). La relación teoría-práctica en la formación permanente del profesorado. Sevilla: Díada.

Stake, R. (1999). Investigación con estudio de casos. España: Morata.

Schön, D. (1987). La formación de profesores reflexivos. Hacia un nuevo diseño de la enseñanza y el aprendizaje. Barcelona: Editorial Paidós.

Stenhouse, L. (1991). Investigación y desarrollo del curriculum. España: Editorial Morata.

Stenhouse, L. (1996). La investigación como base de la enseñanza. España: Editorial Morata. 